\title{
DYNAMIC THERMAL SHOCK IN HOLLOW SPHERES ${ }^{1}$
}

\author{
BY \\ T. A. ZAKER \\ IIT Research Institute, Chicago, Illinois
}

\begin{abstract}
A method is developed for the solution of problems of uncoupled dynamic thermoelasticity with arbitrarily prescribed spherically symmetric temperature fields. The technique is based on an integration theorem for hyperbolic initial-value problems, together with the construction of image temperature fields in regions outside the actual body so as to satisfy stress or displacement boundary conditions at surfaces.

The image technique is applied to problems of internal thermal shock in spherical shells of arbitrary thickness, both with stress-free and with fixed isothermal external boundaries. The published closed-form solution of the associated simpler problem of thermal shock on a cavity in an infinite solid, which had been obtained by other investigators using transform techniques, is recovered as a special case. The results for the cavity and unrestrained shell problems are compared with those obtained by other techniques. It is shown that the image method resolves accurately the details of the dynamic response at early times in shell problems. The problem of the rigidly embedded shell is of interest in connection with determining the far-field signature of a buried radiation burst.
\end{abstract}

$$
\begin{array}{cl} 
& \multicolumn{1}{c}{\text { List of Symbols }} \\
\text { Symbol } & \multicolumn{1}{c}{\text { Definition }} \\
a & =\text { cavity radius } \\
c & =(2 \mu / \rho p)^{1 / 2}, \text { dilatation wave speed } \\
f, g & =\text { image field components } \\
k, \bar{k} & =(-p \pm i q) / \gamma, \text { complex frequencies } \\
p, q & =(1-2 \nu) /(1-\nu),(1-2 \nu)^{1 / 2} /(1-\nu) \\
r, t & =\text { radial position, time } \\
T, u & =\text { temperature, displacement } \\
x, y & =t \pm \gamma(r-1), \text { characteristic variables } \\
\alpha, \kappa & =\text { expansion coefficient, diffusivity } \\
\beta & =\text { shell radius ratio } \\
\gamma & =\kappa / c a, \text { inertia parameter } \\
\mu, \nu & =\text { shear modulus, Poisson's ratio } \\
\rho & =\text { density } \\
\sigma^{(r)}, \sigma^{(\theta)} & =\text { radial, circumferential stresses } \\
\varphi & =\text { displacement potential } \\
\omega & =1 / \gamma \beta .
\end{array}
$$

Except for physical constants, the quantities listed above are dimensionless. Incidental notation is defined where it appears in the text.

1Received January 7, 1968. Based on a doctoral thesis in applied mechanics submitted to Illinois Institute of Technology, Chicago, Illinois. 
I. Introduction. Thermally excited mechanical response of solid materials is of increasing interest in the engineering sciences. In power-generating equipment such as the fast nuclear reactor, in electromagnetic radiation pulse emitters such as the laser, and in certain military applications, materials may be subjected to sudden, large thermal fluxes.

Nonuniform heating of an elastic solid produces thermal stresses that vary with time and position in the material. For sufficiently rapid temperature changes, inertia effects characterized by the propagation of stress waves may be significant.

Few solutions exist for transient thermoelastic problems including inertia effects, owing to the complexity of the governing equations when inertia effects are retained. Except in problems involving propagation of high-frequency mechanical vibration, the effect of inertia can be studied within the framework of uncoupled thermoelasticity theory, in which the effect of rate of volume strain on the transient temperature field is assumed negligible. The temperature field then satisfies a diffusion equation, and can be calculated independently of the mechanical response. This point of view is followed in the present work.

Technically significant inertia effects may occur when rapid power deposition takes place in the bulk of a massive body, as during absorption of electromagnetic energy from a pulsed radiation source. On the other hand, the quasistatic response of bodies to transient thermal stresses brought about by sudden boundary temperature change has been studied extensively, and it is apppropriate to examine the effect of inertia in such problems.

In 1959, Sternberg and Chakravorty [1] solved the dynamic problem of sudden temperature change at the surface of a spherical cavity in an infinite solid within the framework of uncoupled thermoelasticity theory. They obtained an exact, closed-form solution of the displacement equations of motion using transform techniques. The solution contains discontinuities of stress and velocity produced by the instantaneous change of temperature at the cavity boundary at the initial time, and exhibits radiationdamped, thermally induced oscillation of the cavity.

In 1965, Tsui and Kraus [2] presented results for the uncoupled thermoelastic response of a thick-walled spherical shell to internal thermal shock. They also applied transform techniques successfully to the displacement equations of motion. The temperature of the stress-free external shell boundary in this problem is assumed to be maintained for all time at its initial value. Their work does not, however, represent the most natural generalization of the cavity problem.

In 1965, the present author [3] suggested a technique for the solution of dynamic thermoelastic problems in finite, massive bodies. The technique is based on the construction of image temperature fields in regions outside the actual region in positiontime coordinates, in such a way as to satisfy zero-stress or zero-displacement boundary conditions at surfaces. The technique was applied to the problem of heat addition in flat elastic slabs. For this plane, one-dimensional problem the rules for image construction are quite simple and were developed by a purely physical argument.

In what follows, the technique of image temperature fields is extended to spherically symmetric dynamic thermoelastic problems with arbitrarily prescribed heat addition. The cavity problem of Sternberg and Chakravorty is explicitly recovered analytically as a special case. Analytic results are given for early times in the unrestrained shell problem of Tsui and Kraus as a natural generalization of the cavity problem, and 
precise numerical results are presented. Finally, new analytic and numerical results are exhibited for the early-time response of the spherical shell with internal thermal shock, rigidly embedded in an isothermal medium. The physical situation represented by this last problem is that of a thermoelastically responding capsule with sudden internal heat release, embedded in an isothermal medium of relatively much higher mechanical impedance.

II. Spherically symmetric thermoelastic equations. Consider a spherically symmetric problem of uncoupled dynamic linear thermoelasticity with no internal heat sources [4]. Let the dimensionless radial position $r$, time $t$, temperature change $T$ from the initial state, stress components $\sigma$, radial displacement $u$, and scalar potential $\varphi$ be related to the corresponding dimensional variables (denoted by barred symbols) as follows:

$$
\begin{array}{rlrl}
r & =\bar{r} / a & \sigma & =\frac{1-\nu}{(1+\nu) \alpha \bar{T}_{0}} \frac{\bar{\sigma}}{2 \mu} \\
t & =\kappa t / a^{2} & \varphi & =\frac{1-\nu}{(1+\nu) \alpha \bar{T}_{0}} \frac{\bar{\varphi}}{a^{2}} \\
T & =\bar{T} / \bar{T}_{0} & u & =\partial \varphi / \partial r
\end{array}
$$

where $a$ is a characteristic dimension such as the radius of a cavity, $\bar{T}_{0}$ is a characteristic temperature change, $\mu$ and $\nu$ are the shear modulus and Poisson's ratio, and $\alpha$ and $\kappa$ are the thermal expansion coefficient and diffusivity. The material is assumed to be isotropic and homogeneous.

Differential equations. The temperature in uncoupled thermoelasticity with spherical symmetry satisfies the diffusion equation

$$
(r T)_{r r}=(r T)_{t}
$$

while the potential satisfies the wave equation

$$
(r \varphi)_{r r}-\gamma^{2}(r \varphi)_{t \imath}=r T
$$

where subscripts $r, t$ denote partial differentiation, and the inertia parameter $\gamma$ is defined by

$$
\gamma=\frac{\kappa}{c a}=\frac{\kappa}{a}\left[\frac{\rho(1-2 \nu)}{2 \mu(1-\nu)}\right]^{1 / 2}
$$

where $c$ is the dilatation wave speed and $\rho$ is the density.

It is convenient to write the displacement in terms of the product $(r \varphi)$ as follows:

$$
r^{2} u=r(r \varphi)_{r}-(r \varphi) .
$$

The radial normal stress is related to the potential by $^{2}$

$$
p r \sigma^{(r)}=\gamma^{2}(r \varphi)_{t}-2 p(r \varphi)_{r} / r+2 p(r \varphi) / r^{2}
$$

where $p$ is an elastic constant defined by the relation

$$
p=(1-2 v) /(1-\nu) .
$$

The circumferential normal stress is related to the potential by ${ }^{2}$

\footnotetext{
2The stress-potential relations express Hooke's law for a thermoelastic material, using (2) to eliminate $(r \varphi)_{r r}$.
} 


$$
p r \sigma^{(\theta)}=\gamma^{2}(1-p)(r \varphi)_{\imath}+p(r \varphi)_{r} / r-p(r \varphi) / r^{2}-p(r T) .
$$

In terms of characteristic variables defined by

$$
x, y=t \pm \gamma(r-1)
$$

the wave equation (2) becomes

$$
(r \varphi)_{x y}=-(r T) / 4 \gamma^{2} .
$$

Boundary conditions. We will consider the infinite solid with a spherical cavity and shells of arbitrary thickness, with sudden temperature change at the cavity boundary. In these problems the initial state is a state of rest with zero displacement and uniform temperature throughout.

In the case of a cavity the boundary conditions are

$$
\begin{gathered}
T(1, t)=h(t) \\
\sigma^{(r)}(1, t)=0
\end{gathered}
$$

where $h(t)$ is the unit step function. In addition, the temperature and the stress components vanish at infinity. With no loss of generality we also require

$$
\varphi(r, 0)=0 \text {. }
$$

In the case of an unrestrained shell of dimensionless outer radius $\beta$, the boundary conditions are

$$
\begin{aligned}
T(1, t) & =h(t), & T(\beta, t) & =0 \\
\sigma^{(r)}(1, t) & =0, & \sigma^{(r)}(\beta, t) & =0 .
\end{aligned}
$$

As before, we also require

$$
\varphi(r, 0)=0 .
$$

In the case of a rigidly embedded shell the second of equations (11b) is replaced by

$$
u(\beta, t)=0
$$

the other conditions (11) being retained.

The quasistatic formulations of the foregoing problems are obtained by replacing (2) by

$$
(r \varphi)_{r r}=r T+r C_{0}
$$

where $C_{0}$ is a function of time to be determined from the boundary conditions together with a second essential function of time arising from the integration of (13).

III. Method of images. Dynamic thermoelastic problems of the kind considered in this work are mixed boundary-initial value problems of hyperbolic type. An integral representation of solutions originally due to Riemann [5] exists for hyperbolic initialvalue problems. Boundary data in dynamic thermoelastic problems are not suitable for direct application of integration formulas, however. The construction of image temperature fields circumvents this difficulty by converting the problem to a pure initial-value problem for which a solution can be obtained explicitly by application of Riemann's theorem. 
Riemann integral formula. An initial-value problem governed by the elementary nonhomogeneous wave equation

$$
v_{x y}=f
$$

may be solved by application of Riemann's integration theorem. Consider two characteristics intersecting at point $P$ in the plane of characteristic variables $x, y$ (Fig. 1). Values of the dependent variable $v$ and its first derivatives are assumed to be prescribed along a smooth noncharacteristic line which intersects the two characteristics at $A$ and $B$ and is nowhere tangent to a characteristic direction. Then by Riemann's theorem the value of the dependent variable at $P$ is given by

$$
v(P)=\frac{1}{2}[v(A)+v(B)]+\frac{1}{2} \int_{A B}\left(v_{x} d x-v_{y} d y\right)+\iint_{A B P} f d x d y .
$$

For homogeneous initial data equation (15) reduces to

$$
v(P)=\iint_{A B P} f d x d y .
$$

Consider application of these properties to the dynamic thermoelastic problem of an infinite solid with a spherical cavity. There are two distinct regions in the plane of independent variables (Fig. 2). For a field point $P$ in region 0 (Fig. 2a), determination of the potential from Eq. (9) is a pure initial-value problem, and Eq. (16) applies directly in the form

$$
r \varphi=-\frac{1}{4 \gamma^{2}} \iint_{A B P}(r T) d x d y .
$$

On the other hand, determination of the potential at a field point $P$ in region 1 (Fig. 2b) is a mixed boundary-characteristic value problem, since one boundary line $\left(O A^{\prime}\right)$ of the region is timelike and the other $\left(O B^{\prime}\right)$ is characteristic. Integral formulas analogous to (15) for this mixed problem [5] require for their evaluation values of the dependent variable and its first derivatives along $O A^{\prime}$. This information is not explicitly available at the cavity boundary in a dynamic thermoelastic problem. Instead, the

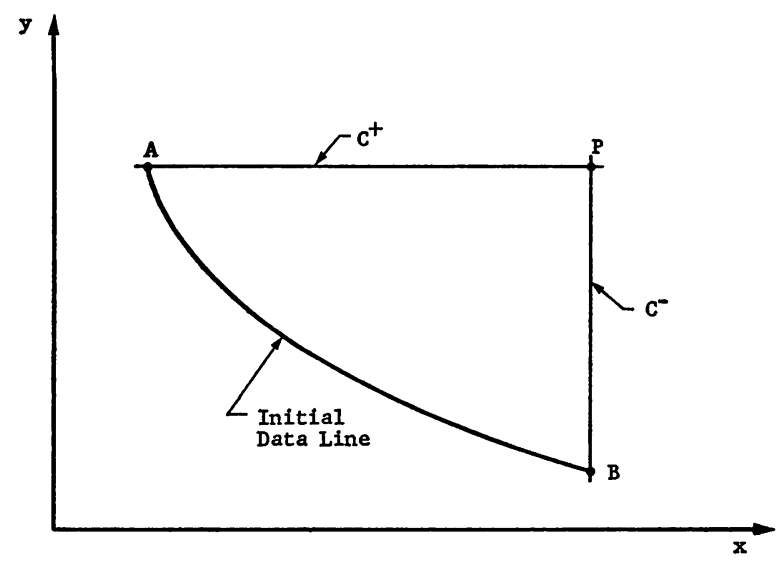

Fig. 1. Hyperbolic initial-value problem. 


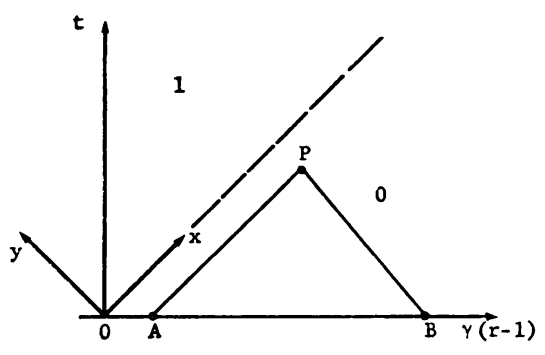

a. For Region 0

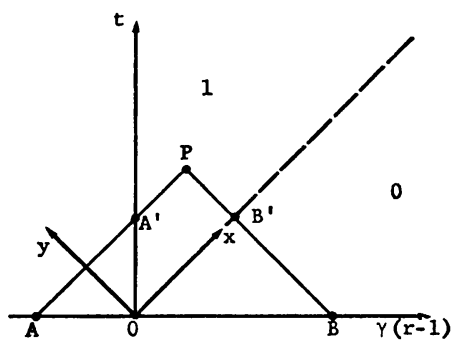

b. For Region 1

Fig. 2. Domains of dependence in a dynamic thermoelastic problem.

boundary condition of zero stress is obtained by setting the right-hand side of (5) equal to zero at $r=1$. This implies a linear relation among derivatives of the potential and the potential itself at the cavity boundary. Similarly, in the case of a fixed boundary at $r=1$, the boundary condition is obtained by setting the right-hand side of (4) equal to zero, implying a linear relation between $(r \varphi)$ and $(r \varphi)_{r}$ there.

By construction of an appropriate image temperature field in $r<1$, the region of integration for a field point $P$ in region 1 of the cavity problem can be expanded to include $A O A^{\prime}$, with homogeneous initial data along $A O$. This converts the determination of the potential in region 1 to a pure initial-value problem for the domain $A B P$, and Eq. (17) then applies as well to any field point $P$ in region 1 . This is the essential feature of the image method.

Image formulas. Consider first the determination of an image temperature field for a stress-free internal (cavity) boundary. Define the variable $f(r-1, t) \equiv r T$ to be given arbitrarily by a known function $f_{0}(r-1, t)$ in $r>1$ and by the function $f_{-1}(r-1, t)$ to be determined in $r<1$. Further define $g_{-1}(r-1, t)$ such that

$$
f_{-1}(r-1, t)=-f_{0}[-(r-1), t]+g_{-1}(r-1, t) .
$$

That is, the image temperature field is the same as the given field reflected in the boundary with reversed algebraic sign as in the case of a plane boundary [3], with an additional component $g_{-1}$ due to the curvature.

The problem is now to determine the image component $g_{-1}$ so that the potential is given by the solution (17) of a pure initial-value problem. In terms of the independent variables $r$ and $t$, Eq. (17) becomes

$-2 \gamma r \varphi=\int_{r-t / \gamma}^{r} \int_{0}^{t-\gamma(r-\xi)} f(\xi-1, \tau) d \tau d \xi+\int_{r}^{r+t / \gamma} \int_{0}^{t+\gamma(r-\xi)} f(\xi-1, \tau) d \tau d \xi$.

The expression for the radial stress is formed from Eq. (5), using $r \varphi$ given by Eq. (19) and applying the usual rules for differentiation of integrals. Requiring the radial stress to be zero at $r=1$ and making use of (18), one reaches the following functional integrodifferential equation for the determination of $g_{-1}$ :

$$
\begin{aligned}
\int_{-t / \gamma}^{0}\left[\gamma^{2} g_{-1}^{\prime}(\zeta, t+\gamma \zeta)+2 p \gamma g_{-1}(\zeta, t+\gamma \zeta)+2 p \int_{0}^{t+\gamma \zeta} g_{-1}(\zeta, \tau) d \tau\right. \\
\left.-4 p \gamma f_{0}(-\zeta, t+\gamma \zeta)\right] d \zeta+\gamma g_{-1}(-t / \gamma, 0)=0
\end{aligned}
$$

where the prime denotes differentiation with respect to the second argument. 
Equation (20) does not determine uniquely the image field component $g_{-1}$, since a boundary condition is lacking. For simplicity we seek a solution of (20) which also satisfies

$$
g_{-1}(-t / \gamma, 0)=0 .
$$

The solution of (20) with the condition (21) can be written

$$
g_{-1}(r-1, t)=-\frac{4 p}{q} \operatorname{Re}\left\{i k \int_{0}^{t} f_{0}[-(r-1), \eta] \exp [k(t-\eta)] d n\right\}
$$

where

$$
q=\left(2 p-p^{2}\right)^{1 / 2}
$$

and $k$ is a complex parameter defined by

$$
\gamma k=-p+i q .
$$

The real and imaginary parts of $k$ correspond respectively to the radiation damping coefficient for outgoing spherical waves and to the natural frequency of cavity oscillation.

Consider next the determination of an image temperature field for a stress-free external boundary of radius $\beta$. Define $f(r-\beta, t) \equiv r T$ to be given by the known func$\operatorname{tion}^{3} f_{0}(r-\beta, t)$ in $r<\beta$, and by the function $f_{1}(r-\beta, t)$ to be determined in $r>\beta$. Further define $g_{1}(r-\beta, t)$ such that

$$
f_{1}(r-\beta, t)=-f_{0}[-(r-\beta), t]+g_{1}(r-\beta, t)
$$

with an interpretation similar to that of (18). Proceeding as in the case of an internal boundary, one obtains

$$
\begin{aligned}
\int_{0}^{t / \gamma}\left[\gamma^{2} g_{1}^{\prime}(\zeta, t-\gamma \zeta)-\frac{2 p \gamma}{\beta} g_{1}(\zeta, t-\gamma \zeta)+\frac{2 p}{\beta^{2}} \int_{0}^{t-\gamma \zeta} g_{1}(\zeta, \tau) d \tau\right. \\
\left.\quad+\frac{4 p \gamma}{\beta} f_{0}(-\zeta, t-\gamma \zeta)\right] d \zeta+\gamma g_{1}(t / \gamma, 0)=0
\end{aligned}
$$

The solution of (26) with the assumed condition $g_{1}(t / r, 0)=0$ is

$$
g_{1}(r-\beta, t)=-\frac{4 p}{q} \operatorname{Re}\left\{\frac{i \bar{k}}{\beta} \int_{0}^{t} f_{0}[-(r-\beta), \eta] \exp [-\bar{k}(t-\eta) / \beta] d \eta\right\}
$$

where $\bar{k}$ is the complex conjugate of the parameter $k$ defined by (24). The complex argument of the exponential in (27) has a positive real part, corresponding to convergence amplification of ingoing spherical waves. Both the amplification and the frequency are inversely proportional to the boundary radius $\beta$ in this case.

Finally, consider the case of a fixed external boundary of radius $\beta$. Define $g_{1}$ in this case such that

$$
f_{1}(r-\beta, t)=f_{0}[-(r-\beta), t]+g_{1}(r-\beta, t) .
$$

That is, the given field $f_{0}$ is reflected in the boundary without change of sign.

${ }^{8}$ For the same given temperature field in $1 \leq r \leq \beta, f_{0}$ is in general a different function of $r-\beta$ than of $r-1$. 
Using (19) and expressing the displacement $u$ by the right-hand side of (4), one obtains, upon setting $u=0$ at $r=\beta$, the integral equation

$$
\int_{0}^{t / \gamma}\left\{\gamma g_{1}(\zeta, t-\gamma \zeta)-\frac{1}{\beta} \int_{0}^{t-\gamma \zeta}\left[2 f_{0}(-\zeta, \tau)+g_{1}(\zeta, \tau)\right] d \tau\right\} d \zeta=0 .
$$

The solution of $(29)$ is

$$
g_{1}(r-\beta, t)=\frac{2}{\gamma \beta} \int_{0}^{t} f_{0}[-(r-\beta), \eta] \exp [(t-\eta) / \gamma \beta] d \eta .
$$

The image field is therefore determined by integration of the given field with a real exponential rather than with an exponential of complex argument as for a stress-free boundary.

Multiple images. As shown in Fig. 2, only two distinct solution regions exist in the cavity problem. On the other hand, the shell problem is comprised of infinitely many distinct regions in radial position-time space, separated by the primary characteristic lines originating from the shell boundaries at the initial time, and by successive reflections of these primary characteristics. This natural division of the problem is shown in Fig. 3, in which the solution regions in the actual zone of the problem (0) are identified systematically by two-digit numbers.

It may be shown, as indicated in Fig. 3, that infinitely many temperature image zones exist to either side of the actual zone. For example, in order to convert the mixed boundary-characteristic value problem in region 21 to a pure initial-value problem, the domain of dependence of a field point $P$ at the boundary of region 21 must extend into the image zone +1 on the right of the actual zone. This means that all of the image field in AOP cannot be determined from the given field in the actual zone alone.

At this stage of the solution, however, the image in zone +1 has already been determined from the field in the actual zone so as to satisfy the right-hand (outer) mechanical boundary condition. Moreover, at this stage of the solution the image field in zone -1 has similarly been constructed so as to satisfy the left-hand (inner) mechanical boundary condition when combined with effects originating from positions in the actual zone symmetrically disposed with respect to that boundary.

We conclude that a second image zone $(-2)$ with a different field must exist on the

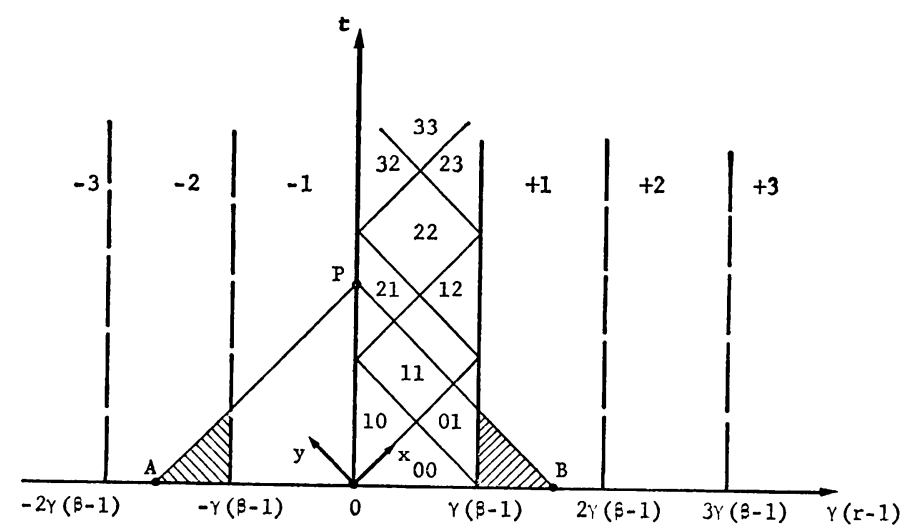

FIG. 3. Image construction in a shell problem. 
left, and that the image zone -1 is equal in thickness to the actual zone. Because at this stage of the solution the field in image zone -1 already satisfies the inner boundary condition, we also conclude that the image field in zone -2 is constructed solely from the known field in zone +1 so as to satisfy the inner boundary condition. In other words, to determine an image formula for zone -2 , one derives the relevant conditions by considering additional effects at the boundary arising out of application of the solution formula (17) only to the shaded regions of Fig. 3. This is evidently a problem equivalent to that of constructing the first image on the left from the given temperature field in the actual zone. Hence, in obvious notation, ${ }^{4}$

$$
\begin{aligned}
& f_{-2}(r-1, t)=-f_{1}[-(r-1), t]+g_{-2}(r-1, t) \\
& g_{-2}(r-1, t)=-\frac{4 p}{q} \operatorname{Re}\left\{i k \int_{0}^{t} f_{1}[-(r-1), \eta] \exp [k(t-\eta)] d \eta\right\}
\end{aligned}
$$

for a stress-free internal boundary.

The foregoing arguments are readily generalized to arbitrarily many solution regions and image zones. At any stage of the solution, integration of the solution formula (17) is performed over a known finite number of image zones. The solution at a point in region $m n$ requires integration extending into $m$ image zones on the left and $n$ on the right in Fig. 3. The index difference $n-m$ has the values $1,0,-1$ respectively for right-boundary regions, central (characteristic-bounded) regions, and left-boundary regions. The required image fields are constructed by repeated application of formulas of the type of (22) and (27) or (30) for an unrestrained shell or an embedded shell.

Solution properties. It is easily established that the solution to the initial-value problem obtained by extending the domain of integration of equation (17) into image zones satisfies the governing equation (9). Since image temperature fields calculated from (22) and (27) or (30) also satisfy the corresponding integral relations (20) and (26) or (29) representing the mechanical boundary conditions, the initial-value problem solution is also the solution of the original dynamic thermoelastic shell problem.

The solution at a typical field point $P$ in radial position-time coordinates is obtained by integration of Eq. (17) over the domain of dependence $A B P$, which in general includes portions of image zones to the left and right of the actual zone. If $r T$ is given by the function $f_{0}$ in the actual zone, one can add to and subtract from the system of fields the same function $f_{0}$ in the image zones. Therefore the solution can always be represented in the form

$$
r \varphi=F_{0}+F_{-} h(y)+F_{+} h[x-\gamma(\beta-1)]
$$

where $F_{0}$ is the result of integrating $f_{0}$ according to Eq. (17) over the entire domain $A B P$, and $F_{-}$and $F_{+}$represent additive contributions from image zones on the left and right. As before, $h$ represents the unit step function. $F_{0}$ is therefore the particular integral of the governing equation which accounts for the right-hand side of (9) but does not satisfy the mechanical boundary conditions. Moreover, in view of the integration limits $x \pm y=$ const. associated with the contributions from image zones, $F_{-}$and $F_{+}$are evidently each functions of one characteristic variable only. Therefore they satisfy (9) with the right-hand side zero, and are complementary solutions of the governing equation.

${ }^{4}$ Here $f_{1}$ is in general a different function of $r-1$ than of $r-\beta$. 
From the foregoing properties, it is evident that the image method is equivalent to finding the particular integral of an uncoupled dynamic thermoelastic problem, and superposing isothermal mechanical loadings at the shell surfaces so as to satisfy boundary conditions. The image method assists in organizing conveniently the analysis of a problem, and in addition provides useful insight into the character of the dynamic response.

IV. Applications. The image method developed in the preceding section was applied to obtain the early dynamic response of shells of arbitrary thickness subjected to internal thermal shock. Sudden internal boundary temperature change and an isothermal external boundary are assumed. Both stress-free (unrestrained shell) and fixed (rigidly embedded shell) external boundary conditions were considered. For times sufficiently early such that the first stress signal from the outer boundary is not yet received at radial positions of interest, the unrestrained shell and embedded shell problems are identical. The associated simpler problem of thermal shock on a spherical cavity in an infinite solid was recovered explicitly as a special case.

Temperature fields. A representation of the solution of (1) with the conditions (11a) exists for the analogous plane problem in terms of a series of complementary error functions. This representation [6] is rapidly convergent at early times. By analogy with the plane problem, the temperature field is given by

$r T=\operatorname{erfc} \frac{r-1}{2(t)^{1 / 2}}+\sum_{m=1}^{\infty}\left[\operatorname{erfc} \frac{2 m(\beta-1)+(r-1)}{2(t)^{1 / 2}}-\operatorname{erfc} \frac{2 m(\beta-1)-(r-1)}{2(t)^{1 / 2}}\right]$

or equivalently by

$r T=\sum_{n=1}^{\infty}\left[\operatorname{erfc} \frac{(2 n-1)(\beta-1)+(r-\beta)}{2(t)^{1 / 2}}-\operatorname{erfc} \frac{(2 n-1)(\beta-1)-(r-\beta)}{2(t)^{1 / 2}}\right]$.

Each error function in either form of the solution satisfies the initial condition and the diffusion equation (1) exactly. Any truncation of (34) satisfies the first of (11a) exactly but the second only approximately. On the other hand, any truncation of (35) satisfies the second of (11a) exactly but the first only approximately. The first term on the right in (34) is the exact solution for the temperature field of the cavity problem.

Images. The dynamic response of unrestrained and rigidly embedded shells was calculated through region 11 of Fig. 3, that is, through $1 \frac{1}{2}$ stress-wave traverses of the shell thickness. To advance the solution to this stage requires the construction of image temperature fields in zone -1 and zone +1 of Fig. 3.

Regions 00 and 10 are unaffected by the external boundary condition. If only the leading term of (34) is retained, the results for these regions comprise the solution of the cavity problem, applicable for all $r \geq 1$ and $t \geq 0$.

To calculate the image component $g_{-1}$ for the stress-free internal shell boundary, Eq. (34), which expresses $f_{0}$ in terms of the argument $r-1$, is used in the formula (21). The result is

$$
\begin{aligned}
g_{-1}(r-1, t)= & -\frac{2 p}{q} \operatorname{Re}\left\{i \exp \left[k^{1 / 2}\left(k^{1 / 2} t+(r-1)\right)\right] \operatorname{erfc}\left(-\frac{r-1}{2(t)^{1 / 2}}-(k t)^{1 / 2}\right)\right. \\
& \left.+i \exp \left[k^{1 / 2}\left(k^{1 / 2} t-(r-1)\right)\right] \operatorname{erfc}\left(-\frac{r-1}{2(t)^{1 / 2}}+(k t)^{1 / 2}\right)\right\}
\end{aligned}
$$




$$
\begin{aligned}
& -\frac{2 p}{q} \sum_{m=1}^{\infty} \operatorname{Re}\left\{i \exp \left[k^{1 / 2}\left(k^{1 / 2} t-R_{-m}\right)\right] \operatorname{erfc}\left(\frac{R_{-m}}{2(t)^{1 / 2}}-(k t)^{1 / 2}\right)\right. \\
& -i \exp \left[k^{1 / 2}\left(k^{1 / 2} t-R_{+m}\right)\right] \operatorname{erfc}\left(\frac{R_{+m}}{2(t)^{1 / 2}}-(k t)^{1 / 2}\right) \\
& +i \exp \left[k^{1 / 2}\left(k^{1 / 2} t+R_{-m}\right)\right] \operatorname{erfc}\left(\frac{R_{-m}}{2(t)^{1 / 2}}+(k t)^{1 / 2}\right) \\
& \left.-i \exp \left[k^{1 / 2}\left(k^{1 / 2} t+R_{+m}\right)\right] \operatorname{erfc}\left(\frac{R_{+m}}{2(t)^{1 / 2}}+(k t)^{1 / 2}\right)\right\}
\end{aligned}
$$

where

$$
R_{ \pm m}=2 \mathrm{~m}(\beta-1) \pm(r-1) .
$$

The terms outside the summation comprise the image field in $r<1$ for the cavity problem, because they arise from the first term on the right in (34). Oscillatory dynamic response may be anticipated, since the image field contains exponentials and error functions of complex arguments, in which $k^{1 / 2}$ stands for the principal value of the root.

To calculate the image component $g_{1}$ for a stress-free external shell boundary, Eq. (35), which expresses $f_{0}$ in terms of the argument $r-\beta$, is used in the formula (27). The result is

$$
\begin{aligned}
g_{1}(r-\beta, t)= & \frac{2 p}{q} \sum_{n=1}^{\infty} \operatorname{Re}\left\{i \exp \left[k^{\prime 1 / 2}\left(k^{\prime 1 / 2} t-R_{-n}\right)\right] \operatorname{erfc}\left(\frac{R_{-n}}{2(t)^{1 / 2}}-\left(k^{\prime} t\right)^{1 / 2}\right)\right. \\
& -i \exp \left[k^{\prime 1 / 2}\left(k^{\prime 1 / 2} t-R_{+n}\right)\right] \operatorname{erfc}\left(\frac{R_{-n}}{2(t)^{1 / 2}}-\left(k^{\prime} t\right)^{1 / 2}\right) \\
& +i \exp \left[k^{\prime 1 / 2}\left(k^{\prime 1 / 2} t+R_{-n}\right)\right] \operatorname{erfc}\left(\frac{R_{-n}}{2(t)^{1 / 2}}+\left(k^{\prime} t\right)^{1 / 2}\right) \\
& \left.-i \exp \left[k^{\prime 1 / 2}\left(k^{\prime 1 / 2} t+R_{+n}\right)\right] \operatorname{erfc}\left(\frac{R_{+n}}{2(t)^{1 / 2}}+\left(k^{\prime} t\right)^{1 / 2}\right)\right\}
\end{aligned}
$$

where

$$
\begin{aligned}
R_{ \pm n} & =(2 n-1)(\beta-1) \pm(r-\beta), \\
k^{\prime} & =-\frac{\bar{k}}{\beta}=\frac{p+i q}{\gamma \beta},
\end{aligned}
$$

$\bar{k}$ being the complex conjugate of the parameter $k$.

Finally, to calculate the image component $g_{1}$ for a fixed external shell boundary, Eq. (35) is used in the formula (30), with the following result:

$$
\begin{aligned}
g_{1}= & -2 \sum_{n=1}^{\infty}\left[\operatorname{erfc} \frac{R_{-n}}{2(t)^{1 / 2}}-\operatorname{erfc} \frac{R_{+n}}{2(t)^{1 / 2}}\right] \\
& +\sum_{n=1}^{\infty}\left\{\exp \left[\omega^{1 / 2}\left(\omega^{1 / 2} t-R_{-n}\right)\right] \operatorname{erfc}\left(\frac{R_{+n}}{2(t)^{1 / 2}}-(\omega t)^{1 / 2}\right)\right. \\
& -\exp \left[\omega^{1 / 2}\left(\omega^{1 / 2} t-R_{+n}\right)\right] \operatorname{erfc}\left(\frac{R_{+n}}{2(t)^{1 / 2}}-(\omega t)^{1 / 2}\right)
\end{aligned}
$$




$$
\begin{aligned}
& +\exp \left[\omega^{1 / 2}\left(\omega^{1 / 2} t+R_{-n}\right)\right] \operatorname{erfc}\left(\frac{R_{-n}}{2(t)^{1 / 2}}+(\omega t)^{1 / 2}\right) \\
& \left.-\exp \left[\omega^{1 / 2}\left(\omega^{1 / 2} t+R_{+n}\right)\right] \operatorname{erfc}\left(\frac{R_{+n}}{2(t)^{1 / 2}}+(\omega t)^{1 / 2}\right)\right\}
\end{aligned}
$$

where $R_{ \pm n}$ are defined by (39), and

$$
\omega=1 / \gamma \beta \text {. }
$$

A remarkable structural similarity exists between image fields $f_{1}$ for the unrestrained and rigidly embedded shells despite the very different external boundary conditions in the two cases. The first summation on the right in (41) is seen to be precisely $-2 f_{0}[-(r-\beta), t]$. In view of (25) and (28), the image fields differ only in that a real parameter $\omega$ in the second summation of (41) replaces the complex parameter $k^{\prime}$ in (38), and the pure imaginary multiplier $2 i p / q$ in (38) is deleted. This similarity, obvious in the complex representation of (38), would be obscured in the equivalent real representation.

Dynamic response. The displacement potentials for the unrestrained and embedded shells are obtained by application of equation (17) to the initial-value problems which result from the construction of images. The solutions in both cases are of the form

$$
r_{\varphi}=F_{0}+F_{-1} h(y)+F_{1} h[x-\gamma(\beta-1)]
$$

in the regions of the problem limited timewise by the characteristics $x=2 \gamma(\beta-1)$ and $y=\gamma(\beta-1)$.

In region 00 of Fig. 3 the solution is independent of the boundary conditions, and can be obtained by integration of either (34) or (35). When the form (34) is used, the solution for the corresponding region of the cavity problem appears explicitly as the leading terms of the result. Thus

$$
\begin{aligned}
F_{0}= & {\left[\frac{(r-1)^{2}}{2}+t+\gamma^{2}\right] \operatorname{erfc} \frac{r-1}{2(t)^{1 / 2}}-(r-1)\left(\frac{t}{\pi}\right)^{1 / 2} \exp \left(-\frac{(r-1)^{2}}{4 t}\right) } \\
& -\frac{\gamma^{2}}{2} \exp \left(\frac{t}{\gamma^{2}}+\frac{r-1}{\gamma}\right) \operatorname{erfc}\left(\frac{r-1}{2(t)^{1 / 2}}+\frac{t^{1 / 2}}{\gamma}\right) \\
& -\frac{\gamma^{2}}{2} \exp \left(\frac{t}{\gamma^{2}}-\frac{r-1}{\gamma}\right) \operatorname{erfc}\left(\frac{r-1}{2(t)^{1 / 2}}-\frac{t^{1 / 2}}{\gamma}\right) \\
& +\sum_{m=1}^{\infty}\left\{\left[\frac{R_{+m}^{2}}{2}+t+\gamma^{2}\right] \operatorname{erfc} \frac{R_{+m}}{2(t)^{1 / 2}}-\left[\frac{R_{-m}^{2}}{2}+t+\gamma^{2}\right] \operatorname{erfc} \frac{R_{-m}}{2(t)^{1 / 2}}\right. \\
& -R_{+m}\left(\frac{t}{\pi}\right)^{1 / 2} \exp \left(-\frac{R_{+m}^{2}}{4 t}\right)+R_{-m}\left(\frac{t}{\pi}\right)^{1 / 2} \exp \left(-\frac{R_{-m}^{2}}{4 t}\right) \\
& -\frac{\gamma^{2}}{2} \exp \left(\frac{t}{\gamma^{2}}+\frac{R_{+m}}{\gamma}\right) \operatorname{erfc}\left(\frac{R_{+m}}{2(t)^{1 / 2}}+\frac{t^{1 / 2}}{\gamma}\right) \\
& +\frac{\gamma^{2}}{2} \exp \left(\frac{t}{\gamma^{2}}+\frac{R_{-m}}{\gamma}\right) \operatorname{erfc}\left(\frac{R_{-m}}{2(t)^{1 / 2}}+\frac{t^{1 / 2}}{\gamma}\right) \\
& -\frac{\gamma^{2}}{2} \exp \left(\frac{t}{\gamma^{2}}-\frac{R_{+m}}{\gamma}\right) \operatorname{erfc}\left(\frac{R_{+m}}{2(t)^{1 / 2}}-\frac{t^{1 / 2}}{\gamma}\right)
\end{aligned}
$$




$$
\left.+\frac{\gamma^{2}}{2} \exp \left(\frac{t}{\gamma^{2}}-\frac{R_{-m}}{\gamma}\right) \operatorname{erfc}\left(\frac{R_{-m}}{2(t)^{1 / 2}}-\frac{t^{1 / 2}}{\gamma}\right)\right\}
$$

where $R_{ \pm m}$ are given by (37). Terms outside the summation in (44) comprise the solution of the cavity problem for $y<0$ (region 0 of Fig. 2).

Additive terms of $r \varphi$ characterizing the response for $y>0$ result from evaluating (17) with the domain of integration extending into the first image zone on the left. One obtains

$$
\begin{aligned}
F_{-1}= & \gamma^{2}\left[\exp \left(\frac{y}{\gamma^{2}}\right)-1\right]-\left(y+2\left(\frac{y}{\pi}\right)^{1 / 2}\right)+\gamma \\
& +\frac{2 p}{q} \operatorname{Re}\left\{\frac { i } { 1 - \gamma ^ { 2 } k } \left[\gamma^{2} \exp \left(\frac{y}{\gamma^{2}}\right) \operatorname{erfc}\left(\frac{y^{1 / 2}}{\gamma}\right)\right.\right. \\
& \left.\left.-\frac{1}{\gamma k^{3 / 2}} \exp (k y) \operatorname{erfc}\left(k^{1 / 2} y\right)+\frac{1-\gamma(k)^{1 / 2}}{\gamma k^{3 / 2}} \exp (k y)\right]\right\} \\
& +2 \sum_{m=1}^{\infty}\left[K_{m} \operatorname{erfc} \frac{K_{m}}{2(y)^{1 / 2}}-2\left(\frac{y}{\pi}\right)^{1 / 2} \exp \left(-\frac{K_{m}^{2}}{4 y}\right)\right] \\
& +\frac{2 p}{q} \sum_{m=1}^{\infty} \operatorname{Re}\left\{\frac { i } { 1 - \gamma ^ { 2 } k } \left[\gamma^{2} \exp \left(\frac{y}{\gamma^{2}}+\frac{K_{m}}{\gamma}\right) \operatorname{erfc}\left(\frac{K_{m}}{2(y)^{1 / 2}}+\frac{y^{1 / 2}}{\gamma}\right)\right.\right. \\
& -\gamma^{2} \exp \left(\frac{y}{\gamma^{2}}-\frac{K_{m}}{\gamma}\right) \operatorname{erfc}\left(\frac{K_{m}}{2(y)^{1 / 2}}-\frac{y^{1 / 2}}{\gamma}\right) \\
& -\frac{1}{\gamma k^{3 / 2}} \exp \left(k y+k^{1 / 2} K_{m}\right) \operatorname{erfc}\left(\frac{K_{m}}{2(y)^{1 / 2}}+(k y)^{1 / 2}\right) \\
& \left.\left.+\frac{1}{\gamma k^{3 / 2}} \exp \left(k y-k^{1 / 2} K_{m}\right) \operatorname{erfc}\left(\frac{K_{m}}{2(y)^{1 / 2}}-(k y)^{1 / 2}\right)\right]\right\}
\end{aligned}
$$

where

$$
K_{m}=2 m(\beta-1) .
$$

Terms outside the summations in (45) comprise the additive component of $r \varphi$ for the cavity problem in $y>0$ (region 1 of Fig. 2).

Additive terms of $r \varphi$ characterizing the response of an unrestrained shell for $x>$ $\gamma(\beta-1)$ result from evaluating (17) with the domain of integration extending into the first image zone on the right. One obtains

$$
\begin{aligned}
F_{1}= & 2 \beta \sum_{n=1}^{\infty}\left[K_{n} \operatorname{erfc} \frac{K_{n}}{2\left(x^{\prime}\right)^{1 / 2}}-2\left(\frac{x^{\prime}}{\pi}\right)^{1 / 2} \exp \left(-\frac{K_{n}^{2}}{4 x^{\prime}}\right)\right] \\
& +\frac{2 p}{q} \sum_{n=1}^{\infty} \operatorname{Re}\left\{\frac { i } { 1 - \gamma ^ { 2 } k ^ { \prime } } \left[\gamma^{2} \exp \left(\frac{x^{\prime}}{\gamma^{2}}+\frac{K_{n}}{\gamma}\right) \operatorname{erfc}\left(\frac{K_{n}}{2\left(x^{\prime}\right)^{1 / 2}}+\frac{x^{\prime 1 / 2}}{\gamma}\right)\right.\right. \\
& -\gamma^{2} \exp \left(\frac{x^{\prime}}{\gamma^{2}}-\frac{K_{n}}{\gamma}\right) \operatorname{erfc}\left(\frac{K_{n}}{2\left(x^{\prime}\right)^{1 / 2}}-\frac{x^{\prime 1 / 2}}{\gamma}\right) \\
& -\frac{1}{\gamma k^{\prime 3 / 2}} \exp \left(k^{\prime} x^{\prime}+k^{\prime 1 / 2} K_{n}\right) \operatorname{erfc}\left(\frac{K_{n}}{2\left(x^{\prime}\right)^{1 / 2}}+\left(k^{\prime} x^{\prime}\right)^{1 / 2}\right) \\
& \left.\left.+\frac{1}{\gamma k^{\prime 3 / 2}} \exp \left(k^{\prime} x^{\prime}-k^{\prime 1 / 2} K_{n}\right) \operatorname{erfc}\left(\frac{K_{n}}{2\left(x^{\prime}\right)^{1 / 2}}-\left(k^{\prime} x^{\prime}\right)^{1 / 2}\right)\right]\right\}
\end{aligned}
$$


where

$$
\begin{aligned}
K_{n} & =(2 n-1)(\beta-1) \\
x^{\prime} & =x-\gamma(\beta-1) .
\end{aligned}
$$

Additive terms of $r \varphi$ characterizing the response of a rigidly embedded shell for $x>\gamma(\beta-1)$ are obtained upon evaluating (17) with the domain of integration extending into the first image zone on the right, where the image field is that for a fixed boundary. The result is

$$
\begin{aligned}
F_{1}= & 2 \beta \sum_{n=1}^{\infty}\left[K_{n} \operatorname{erfc} \frac{K_{n}}{2\left(x^{\prime}\right)^{1 / 2}}-2\left(\frac{x^{\prime}}{\pi}\right)^{1 / 2} \exp \left(-\frac{K_{n}^{2}}{4 x^{\prime}}\right)\right] \\
& +\sum_{n=1}^{\infty}\left\{\frac { 1 } { 1 - \gamma ^ { 2 } \omega } \left[\gamma^{2} \exp \left(\frac{x^{\prime}}{\gamma^{2}}+\frac{K_{n}}{\gamma}\right) \operatorname{erfc}\left(\frac{K_{n}}{2\left(x^{\prime}\right)^{1 / 2}}+\frac{x^{\prime 1 / 2}}{\gamma}\right)\right.\right. \\
& -\gamma^{2} \exp \left(\frac{x^{\prime}}{\gamma^{2}}-\frac{K_{n}}{\gamma}\right) \operatorname{erfc}\left(\frac{K_{n}}{2\left(x^{\prime}\right)^{1 / 2}}-\frac{x^{\prime 1 / 2}}{\gamma}\right) \\
& -\frac{1}{\gamma \omega^{3 / 2}} \exp \left(\omega x^{\prime}+\omega^{1 / 2} K_{n}\right) \operatorname{erfc}\left(\frac{K_{n}}{2\left(x^{\prime}\right)^{1 / 2}}+\left(\omega x^{\prime}\right)^{1 / 2}\right) \\
& \left.\left.+\frac{1}{\gamma \omega^{3 / 2}} \exp \left(\omega x^{\prime}-\omega^{1 / 2} K_{n}\right) \operatorname{erfc}\left(\frac{K_{n}}{2\left(x^{\prime}\right)^{1 / 2}}-\left(\omega x^{\prime}\right)^{1 / 2}\right)\right]\right\}
\end{aligned}
$$

where $K_{n}$ and $x^{\prime}$ are defined as before by (48) and (49). The similarity of structure between (47) and (50) is evident. Again, $k^{\prime}$ in (47) is replaced by the real parameter $\omega$, and the pure imaginary multiplier $2 i p / q$ in the second summation of (47) is deleted.

It is readily verified by differentiation that $F_{0}$ is the particular integral of Eq. (9). Since $F_{-1}$ is a function of $y$ only, and $F_{1}$ for either the unrestrained shell or the embedded shell is a function of $x-\gamma(\beta-1)$ only, they are complementary solutions of (9).

By differentiation of $F_{0}$, one finds that $(r \varphi)_{r}=0$ and $(r \varphi)_{t}=0$ at $t=0$, so that the initial conditions of the problem are satisfied. By appropriate differentiation of the results, substitution into (4) and (5), and evaluation at $r=1$ and $r=\beta$, one finds that the boundary conditions are met.

In all the applications considered in this section, an outgoing discontinuity of stress and velocity propagates along $y=0$, the values of the jumps decreasing as $1 / r$. In the unrestrained shell problem the discontinuity is reflected at the external boundary with reversal of sense, subsequently propagating inward along $x=2 \gamma(\beta-1)$ and increasing as $1 / r$. In the embedded shell problem, the sense of the discontinuity is unchanged upon reflection.

The displacement and the stress components at an arbitrary field point in these problems are formed from (4), (5), and (7) after appropriate differentiation of the foregoing expressions for the potential is carried out. It is found after some rearrangement that the field quantities obtained by transform techniques for the cavity problem [1] are reproduced exactly from the leading terms of the potentials for regions 00 and 10. Moreover, the solution of the cavity problem is expressed more concisely in terms of the potential than in terms of the displacement itself.

On the other hand, only a numerical comparison of results is possible with those of Tsui and Kraus [2] for the unrestrained shell. They applied the Laplace transforma- 
tion to the displacement equation of motion. Transform inversion by residue theory required numerical determination of the poles of the transformed displacement, and the resulting formulas for the displacement are entirely different from those obtainable by differentiation of the foregoing expressions for the potential.

Numerical results. Displacements and stress components were calculated for the infinite solid with a spherical cavity, for the unrestrained shell, and for the rigidly embedded shell for particular values of Poisson's ratio $\nu$ and the inertia parameter $\gamma$. In applications of physical interest, $\gamma$ has values very small compared to unity; for a cavity $1 \mathrm{~cm}$ in diameter in aluminum, for example, $\gamma=2.7 \cdot 10^{-6}$. In order to bring out the qualitative features of the dynamic response and to make comparisons with the results of Sternberg and Chakravorty [1] and of Tsui and Kraus [2], the unrealistically large value of $\gamma=\frac{1}{5}$ was used in the computations, together with the values $\nu=\frac{1}{4}$ for the cavity problem and $\nu=\frac{1}{3}$ for shell problems. Following Tsui and Kraus, an outer-to-inner radius ratio $\beta=2$ was used in the shell computations.

Numerical evaluation of the expressions for the displacement potential and its derivatives was coded in Fortran IV Version 13 programming language for computation on IBM 7094 equipment. This language has a complex arithmetic feature which was used in evaluating the complex representations of oscillatory response associated with stress-free spherical boundaries. It was necessary also to code a subprogram for computation of the complementary error function for arbitrary complex-valued argument. For the radii and times of interest in the shell problems, one term of the summation in (34) beyond the leading term which represents the cavity temperature field, and two terms of the summation in (35), were found to yield sufficient precision in the results. In calculating the temperature with this truncation of (34), the error incurred at the outer boundary (where it is largest), at a time equal to $1 \frac{1}{2}$ wave traverse times, is $5.4 \cdot 10^{-5}$.

Calculations were performed for the cavity problem for $\gamma=\frac{1}{5}$ and $\nu=\frac{1}{4}$. The numerical results for the circumferential stress were found to agree very well with the values reported for this case by Sternberg and Chakravorty [1]. A discontinuity of circumferential stress originates from the cavity boundary at the initial time. The value of the jump is given by

$$
\Delta\left[\sigma^{(\theta)}\right]=(1-p) / p r .
$$

After arrival of this wavefront, the dynamic response is oscillatory in character, representing the effect of cavity vibration induced by thermal shock.

Calculations were performed for unrestrained and rigidly embedded shells for $\beta=2$, $\gamma=\frac{1}{5}$, and $\nu=\frac{1}{3}$, the values used by Tsui and Kraus [2] in their work on the unrestrained shell. The circumferential stress is given as a function of time for these problems at $r=1,1.5$, and 2 in Fig. 4 through Fig. 6 . The corresponding quasistatic and steady-state results are also shown. The two shell problems differ only behind the ingoing characteristic $x=\gamma(\beta-1)$ originating from the outer shell boundary at the initial time. Thus at $r=1$ the dynamic response of the two shells (Fig. 4) is identical as far as it has been calculated; i.e., to $t=\gamma(\beta-1)$.

The present results for the unrestrained shell agree quite well with those of Tsui and Kraus. Moreover, the method used in the present work provides a natural generalization of the cavity problem solution both analytically and computationally. The image method leads to distinct solutions in regions separated by discontinuity lines, 


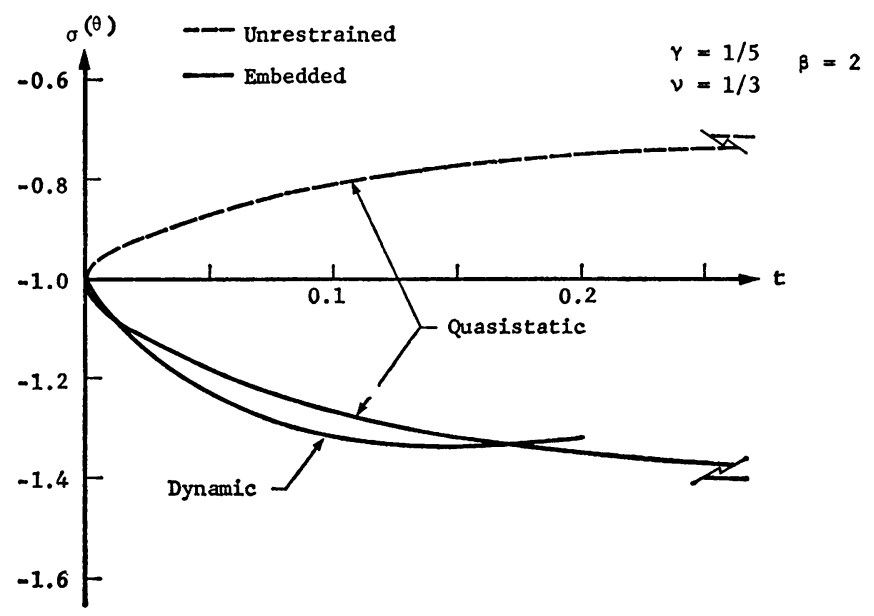

Fig. 4. Circumferential stress in shells, $r=1$.

yielding high precision at early times and positions where inertia effects are most significant.

The discontinuities of stress and velocity, once established by thermal shock in these shell problems, reverberate for all time through the shell thickness, alternately attenuating and amplifying on outgoing and ingoing traverses. Therefore the dynamic

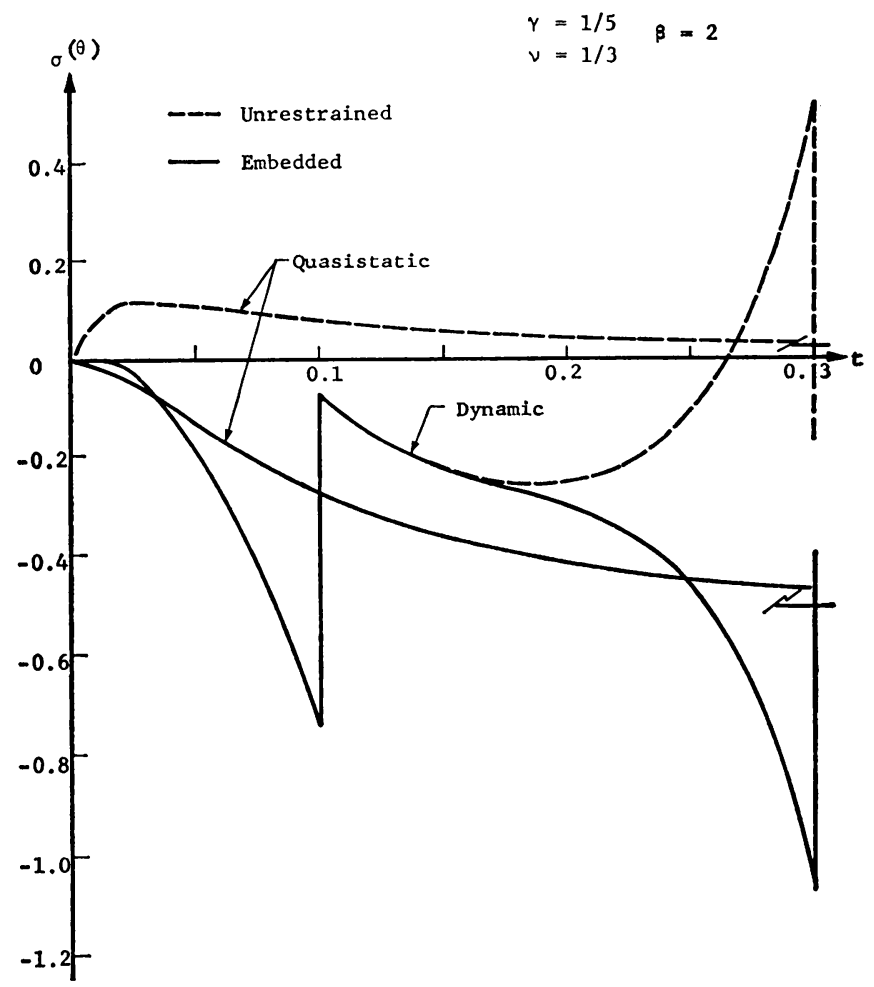

FIG. 5. Circumferential stress in shells, $r=1.5$. 
behavior never strictly approaches quasistatic response. This is in contrast to the cavity problem, in which the discontinuity produced by thermal shock radiates to infinity and the dynamic solution can be shown explicitly to approach the quasistatic and steady-state results.

V. Conclusion. A method has been developed for the solution of problems of uncoupled dynamic thermoelasticity with arbitrarily prescribed spherically symmetric temperature fields. The technique is based on the construction of image temperature fields in regions outside the actual body so as to satisfy stress or displacement boundary conditions at surfaces. This converts the mixed boundary-initial value problem of determining the dynamic response to a pure initial-value hyperbolic problem, to which an integral theorem due to Riemann [5] can be applied explicitly to obtain the solution. $\mathrm{n}$ this respect the image method serves as a useful adjunct to Riemann's integration theorem.

The embedded shell problem may be one of some practical interest in determining the far-field signature of a buried radiation burst. The physical situation represented here is that of a thermoelastically responding capsule with internal heat release, embedded in an isothermal medium of relatively much higher mechanical impedance. The signal radiated to the far field can be calculated according to isothermal elastodynamic theory from the radial stress-time history at the contact surface.

In technically important problems of thermally excited dynamic response, involving heat addition other than by conduction from a heated boundary, it is essential to use a solution method that resolves accurately the fine structure of the dynamic response near discontinuities produced by singularities of the thermal loading. Such a method is provided by the image technique, which reduces problems of this kind to the evaluation of a sequence of finite quadratures. The necessary integrations can be performed numerically with high precision in cases where the temperature fields are prescribed in

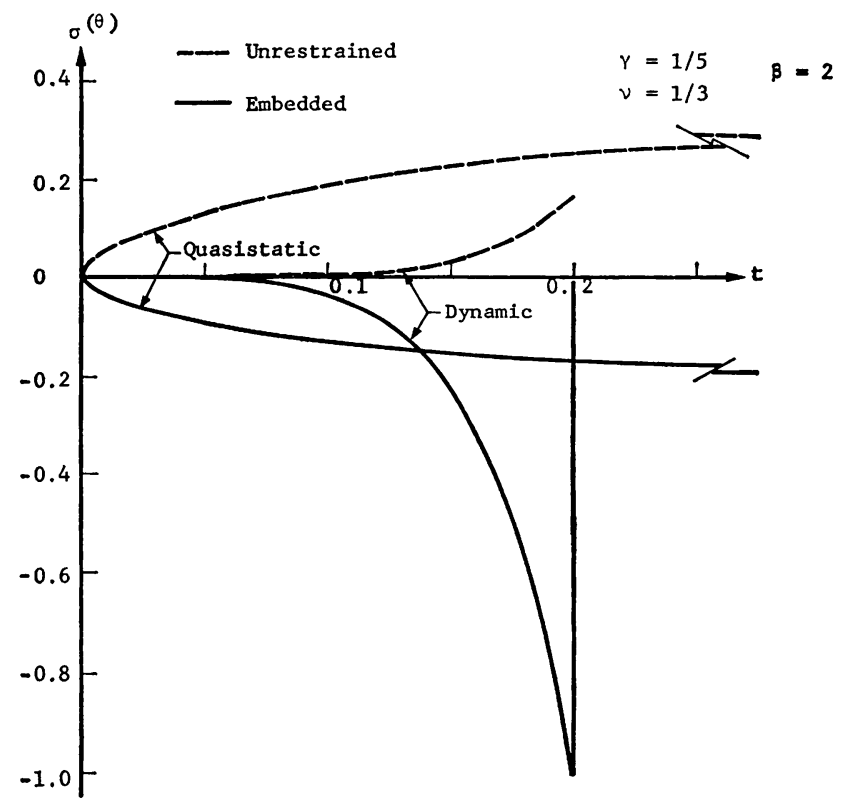

Fia. 6. Circumferential stress in shells, $r=2$. 
nonanalytic form. Image theories can also be developed along the lines of the present work for problems symmetric in other coordinate systems, such as that of thermal shock in thick-walled cylinders.

\section{REFERENCES}

[1] E. Sternberg and J. G. Chakravorty, Thermal shock in an elastic body with a spherical cavity, Quart. Appl. Math. 17, 205-218 (1959)

[2] T. Tsui and H. Kraus, Thermal stress-wave propagation in hollow elastic spheres, J. Acoust. Soc. Amer. 37, 730-737 (1965)

[3] T. A. Zaker, Stress waves generated by heat addition in an elastic solid, J. Appl. Mech. 32, 143-150 (1965)

[4] W. Nowacki, Thermoelasticity, Ch. 4, Addison-Wesley, Reading, Mass., 1962

[5] R. Courant and D. Hilbert, Methods of mathematical physics, Vol. 2, Ch. 5, Interscience, New York, N. Y., 1962

[6] H. S. Carslaw and J. C. Jaeger, Conduction of heat in solids, Ch. 11, Oxford University Press, London, England, 1947 